\section{INTESTINAL STASIS.*}

BY E. G. ECHLESINGER, M.8., B.8., B.sc., LONDON, ENG.

I APPRECIATE very greatly the honor you have done me in asking me to talk to you this evening, and my sole reason for occupying your time is my desire to present to you the views of Mr. Arbuthnot Lane and his school, upon what we consider to be one of the most fundamental problems with which modern medicine and surgery have to deal.

Before considering the general question of intestinal stasis, I would make a few remarks, not directly connected with the subject, but which serve to explain some of the factors at work in producing this widespread condition. Mr. Lane's observations on the skeleton of the coal-heaver and the shoemaker, as well as our everyday experiences in anatomy and surgery, have taught us that the bony and ligamentous skeleton is nothing more nor less than a crystallization of lines of force. Wherever there is a repeated stress or strain, nature deposits along such lines of stress or strain, bony or ligamentous tissue, and such deposits as are of general utility and advantage to the species become inherited as permanent additions to the skeleton. Nature's adaptability to her surroundings, however, is such that a deposit along lines of strain can, and does, occur in the life-time of the individual, as exemplified by the bony bosses on the axis vertebra of the shoemaker and by the evolutionary bands in the abdomen of the constipated individual.

The greatest evolutionary characteristic which distinguishes man from the other animals, is the assumption by him of the erect position. The assumption of this position, while allowing him to evolve at a rapid rate, owing to the free use which it enables him to make of his hands, has not been so happy in its effects on his abdomen, and, further, from the increased mental development which has accompanied the evolution of the arm and hand, there has grown up a series of unnatural and in some senses, degenerative habits, which we know as "civilization." Improper feeding in early life, the continual assumption of an erect attitude and the use of the toilet in defecation contribute to make constipation one of the curses of the race, and one which is even now tending to become a racial inheritance, as well as an individual menace.

What then are the results of constipation, plus the assumption of the erect position, upon the contents of the abdominal cavity? The first portion of the bowel to be affected is the sigmoid. A band develops attaching the sigmoid loop to the side wall of the abdomen, endeavoring both to straighten it out and to prevent the accumulation of material up along the descending colon. This band, if properly developed, is of service, but if over-developed, by hindering the passage

* Read before the Pittsburg Academy of Medicine, April 22, 1913. of matter past it, it increases the condition and a vicious circle is established. The contents of the large gut being damned back, a heavy transverse colon results, the pull of which is felt largely at the flexures. Bands are in consequence deposited, tending to fix these points. Now it is a simple mechanical proposition that, in a hollow tube, if there is a fixed point with a tendency to drop on either side of it, this fixed point forms a potential obstruction, which becomes actual when the dropping forces are in operation. Consequently, in the erect position in the constipated individual, a further obstruction develops at the hepatic and splenic flexures.

Let us now consider the cecum. This organ, when loaded, has a tendency to drop in a downward and inward direction along the wall of the false pelvis. This dropping force produces a strain along lines at right angles to each other, in a direction upwards and inwards, and upwards and outwards respectively. The outer limb of this parallelogram of forces crystallizes as Jackson's membrane, while the inner limb is deposited as a band, which may be attached either to the cecum, to the appendix, or to the ileum, forming, in the latter situation, Lane's kink. If this inner limb fixes itself to the cecum, all is well; if, on the other hand, the appendix or the ileum is the seat of attachment, we have again the fixation of a point in a hollow tube with a tendency to drop on either side, and a potential obstruction at the fixed point. Again, nature may use the appendix as a handy ligament for holding up the cecum and may fix it by its tip to the mesentery of the ileum, a condition which if discovered by the operator is looked upon as evidence of a former appendicitis. The lumen of the terminal ileum is frequently kinked over such a fixed appendix. If the on-flow of the contents of the ileum is delayed, either by a band, or by being held up over a fixed appendix, its contents puddle in its lower coils, which in these cases will be found to occupy the true pelvis.

The beginning of the jejunum passes, as we know, upwards and then downward and to the left from the duodeno-jejunal junction. In an individual, the end of whose ileum is obstructed, the heavy coils of the ileum pull upon this junction, causing it to become an acute angle. Nature, in endeavoring to correct this, forms a band at this point which, while primarily beneficial, unfortunately often increases the angulation. The duodenum, obstructed at its outlet, distends and becomes painful, while its first part, where it is uncovered by peritoneum, frequently shows actual ulceration; in the production of which an ascending infection, of which I shall speak later, is also a factor. Nature discovered the principles of gastro-jejunostomy some time before the surgeon, and endeavored to undo the kink at the duodeno-jejunal junction by forming bands fixing the first part of the jejunum to the under surface of the transverse meso-colon. The obstructed and distended duodenum in- 
duces a spasm of the pylorus, and a consequent stasis of the stomach contents. The heavy stomach, as well as the loaded transverse colon, suspended from the greater curvature, exerts a strain upon the stomach at its line of suspension. This strain is usually felt most on the lesser curvature near the pylorus, and congestion, inflammation, and finally ulceration results.

Now the band which controls the end of the ileum will be found to be attached to the gut at a point opposite to the mesentery, and in contracting, it produces a torsion at the point of attachment, which, super-imposed on the vertical kinking, causes a very real obstruction, often amounting to a 24 hours delay in the passage of a bismuth meal past this point. This delay favors the invasion of the ileal contents by the colonic flora, as well as an excessive growth of the normal flora of the ileum, and this infection may proceed right up the intestines, often ending in an infection of the ducts which open into the gut, with a subsequent production of cholecystitis, gall stones, pancreatitis, etc.

Such then, are the purely mechanical effects of intestinal stasis, but even more striking are the effects of the auto-intoxication which results from the infected ileal contents. A large group of symptoms, which we are in the habit of calling toxic, develops, and which I shall mention in describing the symptomatology of this condition.

Clinically, cases of intestinal stasis fall more or less naturally into four groups, which we may term "the obstructive," the "toxic or suprarenal," the "mixed," and the "end-result", groups. The "obstructive" type is usually regarded as having a duodenal ulcer, a gastric growth, or what is known as "nervous dyspepsia." The patient, more usually a man, complains of pain after food, sometimes at once, sometimes after an interval, with vomiting, which usually relieves the pain. The vomit consists of the food which has lately been eaten, and is quite different from the vomiting which occurs in the second class of cases. "Toxic" symptoms may be present but are often not very marked, and it is not for these that the patient seeks advice. These patients are usually the possessors of apparently thick arteries and have a raised blood-pressure. They usually have quite welldeveloped abdominal muscles. On examination they are very tender over the duodenum and point to this as the site of their pain. They may or may not complain of pain in the iliac fossa, but are invariably extremely tender on pressure in this region, and the end of their ileum is readily palpated as a thick tender cord. A discriminating $\mathrm{x}$-ray examination shows a firmly fixed point in the ileum, and a dilated and hypertrophied duodenum. The effects of ileo-colostomy on this type of case are most striking. The patient can eat and enjoy solid food, when he has often taken nothing but fluids for months; his blood-pressure falls in a few days to near normal, and he can do the hardest and most sustained work with impunity.
It is in this group of cases that paraffin and other non-operative measures are worthless; whilst the operative results are the best, because the ileal and duodenal obstruction is the main feature and brings the patient to the surgeon while the condition of toxemia is yet in its early stages. At the operations on these cases the ileum is found to be hypertrophied proximal to Lane's kink up to five or six times its normal thickness, and the duodenum, which may or may not be ulcerated, is also hypertrophied and distended. Bleeding often takes place from this distended duodenum even without any actual ulceration.

In the obstructive group may be placed many of the cases of so-called Hirchsprung's disease occurring in adults, and some cases of chronic volvulus of the sigmoid.

The second group of cases, the "toxic" or "suprarenal type," occurs more generally in women, and is the group most commonly recognized. In these people constipation is generally present, and, unless it has led to that condition of irritative diarrhea and paralytic secretion commonly called mucous colitis, is often extreme. The direct abdominal symptoms, apart from the constipation, are, however, overshadowed by the group of symptoms called toxic, which are usually regarded as being due to the excessive absorption of bacterial toxins, but for some of which I shall offer another and simpler explanation. These symptoms are both subjective and objective, and are often of such intensity as to make the patient welcome death, since life has become unbearable. Chief among these symptoms is a condition of mental depression and general misery, which is only increased as measure after measure that promises relief is tried, and the condition grows steadily worse. This type of case shows as a rule marked pigmentation, a low blood-pressure, cold and clammy hands and feet and marked general asthenia. Vomiting is practically constant, but differs from the obstructive type in not being related to food and in consisting usually of a watery fluid. Perspiration is often profuse and foul-smelling, moaning headache is present, the skin is harsh and inelastic, and cystic degeneration of the breast is almost invariably present. Pyorrhea alveolaris is often extensive and adds not a little to the wretched condition of the patient.

Cases of this group may have little abdominal pain, but they are tender over the end of the ileum and over the sigmoid, which is straight and readily palpated. X-ray examination in these cases may fail to detect an ileal kink, which indeed may be absent on operation, the patient's natural re-active tendency being in abeyance. This is the extreme picture of this group, but all stages, from an occasional "bilious attack" through the various types of so-called indigestion, neurosis, etc., can be recognized.

The "mixed" group of cases includes those patients who present a mixture of the obstructive and the toxic symptoms. The more marked 
the obstructive, as opposed to the toxic, symptoms are, the better the operative results. The reason of this appears to be simply that the severe abdominal pain of the obstructive type leads them to seek surgical aid much earlier than the patient who, complaining mainly of the misery of "toxemia," has passed through many years of treatments which, while no doubt well meant, and well carried out, unfortunately often have little else to recommend them.

The group to which the term "end result" is applied is a large and varied one, consisting of cases in which the end result has reached such proportions as to over-shadow the underlying cause. This group includes cases of such diverse nature as trigeminal neuralgia, many cases of dementia, of gall-stones, pancreatitis, many cases of cancer of the breast, etc. It also includes a large group of patients in whom the general resistance has been so lowered as the result of intestinal stasis that the body is a ready prey to various infective processes. Under this head come many cases of tuberculosis, rheumatoid arthritis, etc. In this last group it is often a nice question whether the end result has not become too advanced for it to be worth while to deal with the underlying stasis, and of course, in the case of malignant disease, this is out of the question. The boundary of this group of cases is being daily extended, and $I$ feel sure that as our knowledge accumulates, many conditions at present looked on as primary, will be found to depend, in the first instance upon abnormalities in the mechanics, physiology, or bacteriology of the alimentary canal.

The question of how stasis, and the consequent infection of the intestinal contents, produces the symptoms which I have endeavored to describe, is a difficult one to answer, but it appears that four separate factors are at work. In the first place, there are the purely mechanical effects. Secondly, there is an excessive absorption of toxines, whose composition we do not yet know exactly, from the infected alimentary tract. Thirdly, the abnormal organisms in the ileum may invade the blood stream, inducing various auto-infective conditions; and, fourthly, there is, I believe, a chemical factor at work which involves the secretions of many, if not all, of the ductless glands. It appears plain that an internal secretion may be affected in one of two ways: either by disease of the glands producing it or by a deficiency of the precursor from which the secretion is built up.

Animal experiments have shown that the body prefers to metabolize its complex constituents from amino-acids rather than from their ultimate breakdown products. These amino-acids are $a b$ sorbed from the small intestine, and I believe that the infection with the $B$. coli, which results from stasis, is capable of altering materially the amount and nature of the amino-acids absorbed. Tyrosin, for example, is usually regarded as the precursor of adrenalin. Now if the infection of stasis produces a deficient absorption of tyrosin, and we know that the B. coli breaks down tyrosin into phenol and cresol, we can easily understand that a deficient secretion of adrenalin may result. The pigmentation, low blood-pressure, vomiting, and general asthenia of the toxic group of cases is so strikingly suggestive of Addison's disease as to make this theory, at any rate, a suggestive one. I believe that when attention is given to the precursors of the internal secretions, as well as to the glands which build them up, much light will be thrown upon what is, at present, a very dark corner in pathology.

Time will not allow me to discuss the treatment of these conditions fully. Early recognition and proper treatment by an intestinal lubricant, such as liquid paraffin, together with an abdominal support will do much to render operative interference unnecessary. In the presence of a marked Lane's kink and any of the end results of stasis, operative interference is called for and may take the form either of division of the obstructing bands or (and in the majority of cases this will give more satisfactory results) the divided end of the ileum may be implanted into the colon below the last kink.

It is impossible, in a few remarks, to deal with such a subject at all adequately, but I hope that I have, at any rate, said enough to indicate to you the lines along which Mr. Lane and those who agree with him are working, and to give you some idea of the many questions connected with the subject of intestinal stasis.

\section{FIVE GENERATIONS OF BLUE SCLEROT- ICS AND ASSOCIATED OSTEOPOROSIS.}

$$
\text { BY F. A. CONLON, M.D., LAWRENCE, MASS. }
$$

The first mention of a blue sclera that I have been able to find reference to was by Ammon in 1841.

In describing a picture ${ }^{1}$ of "Hydrophlmus," which, by the way, is a very good picture of the hereditary form of blue sclerotics of which I am writing, he says ${ }^{2}$ that this blue colored sclera is also seen in "cyanosis congenita."

Eddowes, ${ }^{3}$ in 1900 , mentions a blue sclera as being present in a boy and in the boy's father.

Peters, ${ }^{4}$ of Rostock, in 1907, was the first to point out its hereditary character, having observed it in four generations of a family.

Since then Stephenson, ${ }^{5}$ Rolleston, ${ }^{6}$ Burrows ${ }^{7}$ and Adair-Dighton ${ }^{8}$ have observed families presenting the same characteristics.

Now this hereditary form of blue sclerotics is not to be confounded with the slight blue tint of the sclera noticed in young children and in certain cases of pronounced anemia. Most writers describe it as a "porcelain blue." Stephenson, however, describes the sclera as having a "leaden appearance." 